University of Wollongong

Research Online

Faculty of Engineering and Information

Faculty of Engineering and Information

Sciences - Papers: Part A

Sciences

$1-1-2014$

\title{
Simultaneous measurement of vibration and parameters of a semiconductor laser using self-mixing interferometry
}

Yan Gao

University of Wollongong, yg904@uowmail.edu.au

Yanguang Yu

University of Wollongong, yanguang@uow.edu.au

Jiangtao Xi

University of Wollongong, jiangtao@uow.edu.au

Qinghua Guo

University of Wollongong, qguo@uow.edu.au

Follow this and additional works at: https://ro.uow.edu.au/eispapers

Part of the Engineering Commons, and the Science and Technology Studies Commons

Research Online is the open access institutional repository for the University of Wollongong. For further information contact the UOW Library: research-pubs@uow.edu.au 


\title{
Simultaneous measurement of vibration and parameters of a semiconductor laser using self-mixing interferometry
}

\author{
Abstract \\ Laser diode (LD)-based self-mixing interferometry (SMI) is a promising technique for noncontact sensing \\ and its instrumentation. According to the well-known Lang-Kobayashi equations, an SMI waveform is \\ shaped by multiple parameters, including linewidth enhancement factor (denoted as $\alpha$ ), optical feedback \\ level factor (denoted as C), and the movement of the external cavity of an LD. This paper presents a new \\ algorithm for simultaneously retrieving the multiple parameters from a piece of SMI signal. First, a set of \\ linear equations is derived based on the existing SMI model. By careful selection of data samples, the \\ linear equations canbe made independent and used todetermine a set of variables, and thus the values of \\ $\alpha$ and $\mathrm{C}$, as well as the reconstruction coefficients of the external movement. The work in this paper lifts \\ the restrictions existing in SMI-based sensing methods, such as prerequisite knowledge of either $\alpha$ and $C$ \\ or vibration information of an external target. Simulations and experiments are conducted to verify the \\ proposed algorithm.

\section{Disciplines} \\ Engineering | Science and Technology Studies

\section{Publication Details} \\ Y. Gao, Y. Yu, J. Xi \& Q. Guo, "Simultaneous measurement of vibration and parameters of a semiconductor \\ laser using self-mixing interferometry," Applied Optics, vol. 53, (19) pp. 4256-4263, 2014.
}




\title{
Simultaneous measurement of vibration and parameters of a semiconductor laser using self- mixing interferometry
}

\author{
Yan Gao, Yanguang Yu, Jiangtao Xi, and Qinghua Guo \\ School of Electrical, Computer and Telecommunications Engineering, University of Wollongong-Northfields Ave, \\ Wollongong, NSW, 2522, Australia \\ *Corresponding author: yanguang@uow.edu.au
}

Received Month April, 2014; revised Month May, 2014; accepted Month X, XXXX; posted Month X, XXXX (Doc. ID XXXXX); published Month X, XXXX

\begin{abstract}
A laser diode (LD) based self-mixing interferometry (SMI) is a promising technique for non-contact sensing and its instrumentation. According to the well-known Lang-Kobayashi (L-K) equations, an SMI waveform is shaped by multiple parameters, including linewidth enhancement factor (denoted as $\alpha$ ), optical feedback level factor (denoted as $C$ ) and the movement of the external cavity of an LD. This paper presents a new algorithm for simultaneously retrieving the multiple parameters from a piece of SMI signal. Firstly, a set of linear equations is derived based on the existing SMI model. By careful selection of data samples, the liner equations can be made independent and used to determine a set of variables, and thus the values of $\alpha$ and $C$, as well as the reconstruction coefficients of the external movement. The work in this paper lifts the restrictions existing in SMI based sensing methods, e.g. requiring pre-requisite knowledge of either $\alpha$ and $C$ or vibration information of an external target. Simulations and experiments are conducted to verify the proposed algorithm. (C) 2014 Optical Society of America

OCIS codes: (120.3180) Interferometry; (280.3420) Laser sensor; (140.5960) Semiconductor laser http://dx.doi/org/10.1364/AO.99.099999
\end{abstract}

\section{Introduction}

Self-mixing interferometry (SMI) has been an active area of research as an enabling technique for non-contact sensing and its instrumentation in recent decades. The basic structure of an SMI consists of a laser diode (LD), a focusing lens and a vibrating target which forms the external cavity of the $\mathrm{LD}$. When a portion of light emitted by the LD is backscattered or reflected by the vibrating external target and re-enters the laser cavity, a modulated lasing field will be formed in both amplitude and phase. The modulated laser power, detected by a photodiode (PD) packaged at the rear facet of $\mathrm{LD}$, is called an SMI signal. It carries the information of the external target vibration and the parameters related to the $\mathrm{LD}$. Generally, there are two classes of applications for SMI based sensing: 1) estimation of parameters associated with an $\mathrm{LD}$; and 2) measurement of the metrological quantities of the external target.

Use of SMI for measuring LD parameters mainly focuses on the linewidth enhancement factor and the optical feedback level [1-8]. The linewidth enhancement factor, also called the alpha factor $(\alpha$-parameter $)$, is one of fundamental parameters in the $\mathrm{LDs}$ as it characterizes the $\mathrm{LD}$ linewidth, the chirp, the injection lock range and the response to optical feedback. The optical feedback level, denoted as $C$, describes the coupling strength of the optical feedback in the LD cavity and is utilized for classifying different optical feedback regimes, namely weak feedback regime (where $0<C<1$ ), moderate feedback regime (where $1<C<4.6$ ) and strong feedback regime (where $C>4.6$ ). The existing methods in this application class have the following limitations: 1) The SMI system has to operate in a certain feedback range, e.g. the methods in $[2,4,8]$ are valid for weak feedback regime ( $0<C<1)$ and the method in [1] can be used for moderate regime $(1<C<3) ; 2)$ a prior knowledge is required on the movement of the external cavity $[2,4,8]$, e.g., the movement must be in the form of a simple harmonic vibration; 3) complex signal processing techniques are required such as phase unwrapping (PUM) in [5, 7] and data-to-model fitting algorithms in $[2,4,8]$. These requirements bring a certain difficulty to implement practical systems. Therefore, it is of importance to have a method that can be used without the above restrictions on the optical feedback regime and the external target vibration.

Another class of applications is on the measurement of a target movement. The resolution of SMI based measurement using the fringe counting method is half-wavelength. The resolution can be greatly improved by sophisticated signal pre-processing, fringe sub-division and SMI model-based reconstruction. However, all existing approaches suffer from some restrictions which may degrade the performance. In 1995, a fringe counting method [9] was developed based on the theory that each fringe on an SMI signal represents a half-wavelength shift of a vibrating target. The method is easy to implement but the resolution is only a 
half-wavelength for vibration measurement. In order to improve the accuracy of the vibration measurement, different methods have been developed [5, 10-15], but all valid to a certain range of $C$. The methods in $[9,12,14]$ can only be applied in moderate regime where $1<C<4.6$ and the method in [11] is only valid in weak regime where $0<C<1$. The method in [15] can work in both weak and moderate feedback regimes. At the weak feedback, the fringe counting (with half wavelength resolution) is used for vibration detection. At the moderate feedback, similar to [14], each SMI fringe is treated as a linear sawtooth shape, ignoring the influence of parameters $C$ and $\alpha$ on fringe shape, which causes detection errors on vibration. Besides, the accurate values for $\alpha$ and $C$ are pre-required by methods [5, 9, 10], which is not always possible in practice. Moreover, computationally extensive operations are also required by these methods, such as PUM [5, 10, 12], interpretation of interfringe of SMI signal [11] and Evolutionary algorithm [13], which can degrade the efficiency of these approaches.

The purpose of this paper is to present a novel algorithm that is able to simultaneously retrieve vibration information and $\mathrm{LD}$ related parameters, thus lifting the restrictions on all existing techniques in literature. The proposed method is also characterized by simple computation, thus making it easy to be implemented in practice.

\section{Theory and algorithm}

\section{A. SMI model}

The basic theoretical model for an SMI system is shown as follows [2].

$$
\begin{aligned}
& p(t)=p_{0}[1+m \cdot g(t)] \\
& g(t)=\cos \left(\phi_{F}(t)\right) \\
& \phi_{0}(t)=\phi_{F}(t)+C \cdot \sin \left[\phi_{F}(t)+\arctan (\alpha)\right] \\
& \phi_{0}(t)=\frac{4 \pi}{\lambda_{0}}\left[L_{0}+\Delta L(t)\right]
\end{aligned}
$$

The physical meanings of the symbols in the above model are summarized in table 1.

Above SMI model shows that an SMI signal, that is $g(t)$, carries the information of the external cavity movement and the parameters $\alpha$ and $C$. In the following, we aim to simultaneously retrieve all of these parameters. Note that $\phi_{0}(t)$ can also be considered as the displacement due to its proportional relationship to $\Delta L(t)$.

\section{Table 1. Physical meaning of symbols in Equation (1)-(4)}

\begin{tabular}{c|c}
\hline \hline Symbol & Physical meaning \\
\hline$t$ & Time index \\
$p_{0}$ & Output intensity emitted by free running LD \\
$p(t)$ & Laser output \\
$m$ & Modulation index (typically $\left.m \approx 10^{-3}\right)$ \\
$\phi_{0}(t)$ & Light phase of free running LD \\
$\phi_{F}(t)$ & Light phase of LD with optical feedback \\
$g(t)$ & Normalized laser output, also called SMI signal \\
$C$ & Optical feedback level \\
$\alpha$ & Linewidth enhancement factor \\
$L_{0}$ & Initial external cavity length \\
$\Delta L(t)$ & Displacement of the external target \\
$\lambda_{0}$ & Emitting wavelength of the free running $\mathrm{LD}$ \\
\hline \hline
\end{tabular}

\section{B. New algorithm}

Supposing that the external target is in a periodical vibration, that is, $\Delta L(t)$ is periodic with a fundamental frequency of $\omega_{0}$. From Eq. (4), $\phi_{0}(t)$ is also periodic and can be expressed in the form of Fourier series as below:

$$
\phi_{0}(t) \approx \frac{4 \pi}{\lambda_{0}} L_{0}+\sum_{k=1}^{N}\left[a_{k} \cos \left(k \omega_{0} t\right)+b_{k} \sin \left(k \omega_{0} t\right)\right]
$$

where $k$ is an integer number. $N$ represents $N$ th order Fourier series expansion. $a_{k}$ and $b_{k}$ are the coefficients in Fourier series.

Taking derivative on Eq. (5) with respect to time index $t$, we have:

$$
\frac{d \phi_{0}(t)}{d t}=\omega_{0} \sum_{k=1}^{N}\left[b_{k} k \cos \left(k \omega_{0} t\right)-a_{k} k \sin \left(k \omega_{0} t\right)\right]
$$

Then taking derivative on Eq. (2) and Eq. (3), we have:

$$
\begin{gathered}
\frac{d g(t)}{d t}=\frac{d \phi_{F}(t)}{d t}\left[-\sin \left(\phi_{F}(t)\right)\right] \\
\frac{d \phi_{0}(t)}{d t}=\frac{d \phi_{F}(t)}{d t}+C \frac{d \phi_{F}(t)}{d t} \cos \left[\phi_{F}(t)+\arctan (\alpha)\right]
\end{gathered}
$$

Eq. (7) can be written as below:

$$
\left.\frac{d \phi_{F}(t)}{d t}=-\frac{d g(t) / d t}{\sin \left(\phi_{F}(t)\right)}, \quad\left(\text { when } \sin \left(\phi_{F}(t)\right) \neq 0\right)\right)
$$

For the simplicity of illustration, we use $g^{\prime}(t)$ to replace $d g(t) / d t$ in following.

Substituting Eqs. (6) and (9) into (8), we have: 


$$
\omega_{0} \sum_{k=1}^{N}\left[b_{k} k \cos \left(k \omega_{0} t\right)-a_{k} k \sin \left(k \omega_{0} t\right)\right]=\left[1+C \cos \left(\phi_{F}(t)+\arctan (\alpha)\right)\right]\left(-\frac{g^{\prime}(t)}{\sin \left(\phi_{F}(t)\right)}\right)
$$

as

$$
\begin{aligned}
\sin \left(\phi_{F}(t)\right) & =\left\{\begin{array}{l}
+\sqrt{1-\cos ^{2}\left(\phi_{F}(t)\right)} \\
-\sqrt{1-\cos ^{2}\left(\phi_{F}(t)\right)}
\end{array}\right. \\
& = \begin{cases}+\sqrt{1-g^{2}(t)}, & \text { when } g^{\prime}(t)<0 \\
-\sqrt{1-g^{2}(t)}, & \text { when } g^{\prime}(t)>0\end{cases}
\end{aligned}
$$

Therefore, Eq. (10) can be written as:

$$
\left\{\begin{array}{r}
\omega_{0} \sum_{k=1}^{N}\left[b_{k} k \cos \left(k \omega_{0} t\right)-a_{k} k \sin \left(k \omega_{0} t\right)\right]=\left[1+C \cos (\arctan (\alpha)) g(t)-C \sin (\arctan (\alpha)) \sqrt{1-g^{2}(t)}\right]\left(\begin{array}{r}
g^{\prime}(t) \\
\sqrt{1-g^{2}(t)}
\end{array}\right) \\
\left(\text { when } g^{\prime}(t)<0\right) \\
\omega_{0} \sum_{k=1}^{N}\left[b_{k} k \cos \left(k \omega_{0} t\right)-a_{k} k \sin \left(k \omega_{0} t\right)\right]=\left[1+C \cos (\arctan (\alpha)) g(t)-C \sin (\arctan (\alpha))\left(-\sqrt{1-g^{2}(t)}\right)\right]\left(\begin{array}{c}
\left.-\frac{g^{\prime}(t)}{\left(-\sqrt{1-g^{2}(t)}\right)}\right) \\
\left(\text { when } g^{\prime}(t)>0\right)
\end{array}\right.
\end{array}\right.
$$

Re-arranging Eq. (12) yields the following:

$$
\left\{\begin{array}{rlr}
g^{\prime}(t) & =-g^{\prime}(t) g(t) u_{1}+g^{\prime}(t) \sqrt{1-g^{2}(t)} u_{2} \\
& +\omega_{0} \sqrt{1-g^{2}(t)} \sum_{k=1}^{N} a_{k} k \sin \left(k \omega_{0} t\right)-\omega_{0} \sqrt{1-g^{2}(t)} \sum_{k=1}^{N} b_{k} k \cos \left(k \omega_{0} t\right) & \\
g^{\prime}(t) & =-g^{\prime}(t) g(t) u_{1}-g^{\prime}(t) \sqrt{1-g^{2}(t)} u_{2} & \\
& -\omega_{0} \sqrt{1-g^{2}(t)} \sum_{k=1}^{N} a_{k} k \sin \left(k \omega_{0} t\right)+\omega_{0} \sqrt{1-g^{2}(t)} \sum_{k=1}^{N} b_{k} k \cos \left(k \omega_{0} t\right) & ,\left(\text { when } g^{\prime}(t)<0\right)
\end{array}\right.
$$

where

$$
\left\{\begin{array}{l}
u_{1}=C \cos (\arctan (\alpha)) \\
u_{2}=C \sin (\arctan (\alpha))
\end{array}\right.
$$

From Eq. (14) we have:

$$
\left\{\begin{array}{l}
C=\sqrt{u_{1}^{2}+u_{2}^{2}} \\
\alpha=\frac{u_{2}}{u_{1}}
\end{array}\right.
$$

In Eq. (13), $g(t)$ and $g^{\prime}(t)$ are sample data (constant) at a certain time instance t obtained from an observed SMI signal. Other parameters, including $u_{1}, u_{2}, a_{k}$ and $b_{k}$ are to be estimated shown as below.

We introduce

$$
\begin{aligned}
& G_{1}(t)=g^{\prime}(t) g(t) \\
& G_{2}(t)=g^{\prime}(t) \sqrt{1-g^{2}(t)} \\
& G_{3}(t)=g^{\prime}(t) \\
& A_{k}(t)=\omega_{0} \sqrt{1-g^{2}(t)} k \sin \left(k \omega_{0} t\right), \quad(\text { where } k=1: N) \\
& B_{k}(t)=\omega_{0} \sqrt{1-g^{2}(t)} k \cos \left(k \omega_{0} t\right), \quad(\text { where } k=1: N)
\end{aligned}
$$

Note that $\omega_{0}$ is treated as a known parameter, as it can be easily obtained by applying an auto-correlation operation on a piece of SMI signal [2]. Hence, the five quantities introduced in Eq. (16) are all constant at a certain time instance $t$. With the notation in Eq. (16), Eq. (13) can be written as follows: 


$$
\left\{\begin{array}{r}
-G_{1}(t) u_{1}+G_{2}(t) u_{2}+\sum_{k=1}^{N} A_{k}(t) a_{k}-\sum_{k=1}^{N} B_{k}(t) b_{k}=G_{3}(t) \\
\left(\text { when } g^{\prime}(t)<0\right) \\
-G_{1}(t) u_{1}-G_{2}(t) u_{2}-\sum_{k=1}^{N} A_{k}(t) a_{k}+\sum_{k=1}^{N} B_{k}(t) b_{k}=G_{3}(t) \\
\left(\text { when } g^{\prime}(t)>0\right)
\end{array}\right.
$$

Obviously, Eq. (17) is a linear equation of constant coefficients with $u_{1}, u_{2}, a_{k}$ and $b_{k}$ as variables. As Eq. (17) has $2 N+2$ variables, we need the same number of independent equations. To achieve this we take $2 N+2$ samples from the observed $g(t)$ and its corresponding derivative version $g^{\prime}(t)$, enabling us to have the following:

$\left[\begin{array}{cccccccc}-G_{1}(1) & G_{2}(1) & A_{1}(1) & \cdots & A_{N}(1) & -B_{1}(1) & \cdots & -B_{N}(1) \\ -G_{1}(2) & G_{2}(2) & A_{1}(2) & \cdots & A_{N}(2) & -B_{1}(2) & \cdots & -B_{N}(2) \\ \vdots & \vdots & \vdots & \vdots & \vdots & \vdots & \vdots & \vdots \\ -G_{1}(\mathrm{~N}+1) & G_{2}(\mathrm{~N}+1) & A_{1}(\mathrm{~N}+1) & \cdots & A_{N}(\mathrm{~N}+1) & -B_{1}(\mathrm{~N}+1) & \cdots & -B_{N}(\mathrm{~N}+1) \\ -G_{1}(\mathrm{~N}+2) & -G_{2}(\mathrm{~N}+2) & -A_{1}(\mathrm{~N}+2) & \cdots & -A_{N}(\mathrm{~N}+2) & B_{1}(\mathrm{~N}+2) & \cdots & B_{N}(\mathrm{~N}+2) \\ \vdots & \vdots & \vdots & \vdots & \vdots & \vdots & \vdots & \vdots \\ -G_{1}(2 \mathrm{~N}+2) & -G_{2}(2 \mathrm{~N}+2) & -A_{1}(2 \mathrm{~N}+2) & \cdots & -A_{N}(2 \mathrm{~N}+2) & B_{1}(2 \mathrm{~N}+2) & \cdots & B_{N}(2 \mathrm{~N}+2)\end{array}\right]\left[\begin{array}{c}u_{1} \\ u_{2} \\ a_{1} \\ \vdots \\ a_{N} \\ b_{1} \\ \vdots \\ b_{N}\end{array}\right]=\left[\begin{array}{c}G_{3}(1) \\ G_{3}(2) \\ \vdots \\ G_{3}(\mathrm{~N}+1) \\ G_{3}(\mathrm{~N}+2) \\ \vdots \\ G_{3}(2 \mathrm{~N}+2)\end{array}\right]$

In order to determine all variables $u_{1}, u_{2}, a_{k}$ and $b_{k}$, we must guarantee the Eq. (18) is nontrivial by proper selection of the data samples from the SMI signal to construct above matrix equation. Generally speaking, the larger the differences in the values and the time instances taken among the data samples, the less likely for Eq. (18) to become trivial. Hence, we try to choose the data samples carefully and the sample data will be selected based on the following:

1. The segment of SMI signal corresponding to a whole period of vibration usually consists of two regions corresponding to the increasing and decreasing part of the $\phi_{0}(t)$ respectively. $N+1$ data samples will be taken from the $g^{\prime}(t)<0$ region and the other $N+1$ samples will be from the $g^{\prime}(t)>0$ region (shown in Fig. 1).

2. Assuming that there are $M$ fringes in $g^{\prime}(t)<0$ region in one segment of SMI signal, we set $N+1$ equally spaced levels over the fringe peak-to-peak height, yielding $N+1$ sample points within each individual $g^{\prime}(t)<0$ section.

3. Within each fringe, for the $g^{\prime}(t)<0$ section, $(N+1) / M$ samples are selected, resulting in total number of $N+1$ data samples distributed over the entire $g^{\prime}(t)<0$ region.

4. The other $N+1$ samples are selected in the same way from the region where $g^{\prime}(t)>0$.
Note that, For a target with reciprocating vibration, the same number of fringes will appear in the increasing/decreasing part of $\phi_{0}(t)$. However, the proposed algorithm itself does not require same fringe number for both parts.

Let us use an example to demonstrate the procedures described above. Fig. 1 (a) shows the signal $\phi_{0}(t)$ corresponding to the movement of the external target. Fig. 1 (c) and (e) show the SMI signals $g(t)$ in moderate and weak feedback regimes respectively. Fig. $1(\mathrm{~b})$ and $(\mathrm{d})$ show the derivative forms $\left(g^{\prime}(t)\right)$ of the SMI signals in (c) and (e). The signals shown in Fig.1 are generated using the model described in Eqs. (1)-(5) with parameters $C / \alpha=3.000 / 3.000$ for moderate feedback case and $C / \alpha=0.800 / 3.000$ for weak feedback. Assuming it is accurate enough for a vibration $\phi_{0}(t)$ to be described by the 4 th-order Fourier series, that is:

$$
\begin{aligned}
\phi_{0}(t)= & 8 \times \cos (2 \pi \times 100 t)+2 \times \cos (4 \pi \times 100 t) \\
& +1 \times \cos (6 \pi \times 100 t)+0.5 \times \cos (8 \pi \times 100 t) \\
& +8 \times \sin (2 \pi \times 100 t)+2 \times \sin (4 \pi \times 100 t) \\
& +1 \times \sin (6 \pi \times 100 t)+0.5 \times \sin (8 \pi \times 100 t)
\end{aligned}
$$

The Fig. 1 (b)-(e) illustrate how the data samples are selected. Both $g(t)$ and $g^{\prime}(t)$ will be used in Eq. (16) to obtain coefficients of Eq. (18). By solving Eq. (18) we will be able to get all the variables $\left[\begin{array}{llllllll}u_{1} & u_{2} & a_{1} & \cdots & a_{N} & b_{1} & \cdots & b_{N}\end{array}\right]^{T}$, and finally we can use Eq. (15) to determine $\alpha$ and $C$. 


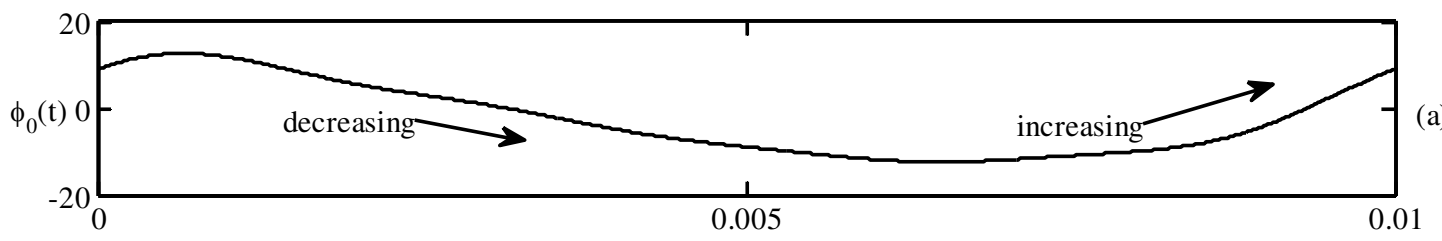

(a)
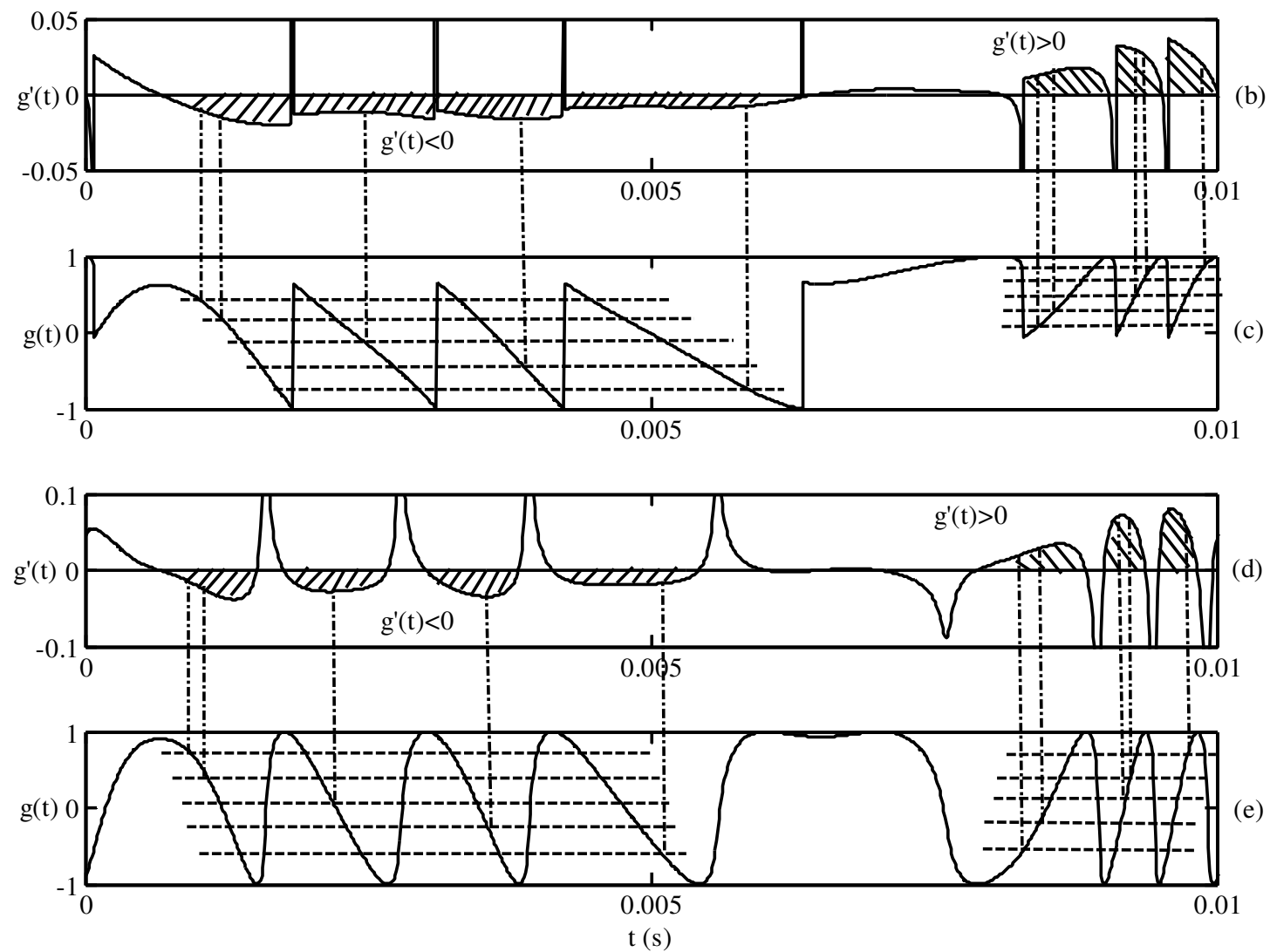

Fig. 1. Illustration of data selection. (a) phase $\phi_{0}(t)$ corresponds to a period of external vibration; (b) Derivative form ( $\left.g^{\prime}(t)\right)$ of the SMI signal $g(t)$ in (c); (c) A SMI signal $g(t)$ in moderate feedback regime; (d) Derivative form $\left(g^{\prime}(t)\right)$ of the SMI signal $g(t)$ in (e); (e) A SMI signal $g(t)$ in weak feedback regime;

\section{Verification}

\section{A. Simulation}

In order to verify the proposed algorithm, we firstly use the simulation data generated by the SMI model described in Eqs. (1)-(5) and apply the proposed algorithm on the data. We consider that the external target vibrates in a triangular form. Therefore, its corresponding $\phi_{0}(t)$ has a peak-to-peak amplitude of 60.000 (rad) and fundamental frequency of $100 \mathrm{~Hz}$ shown in Eq. (19).

$$
\phi_{0}(t)= \begin{cases}30-\frac{60}{T_{0}} t, & \text { when } 0 \leq \mathrm{t}<\frac{T_{0}}{2} \\ 30+\frac{60}{T_{0}} t, & \text { when } \frac{T_{0}}{2} \leq \mathrm{t}<\mathrm{T}_{0}\end{cases}
$$

In general, the higher order of Fourier Series we use, the better reconstruction we can obtain. In this simulation, the Fourier expansion with 10th-order is sufficient to express a triangular vibration if there is no sharp change occurring on the movement. Hence, we expressed the $\phi_{0}(t)$ in Eq. (20):

$$
\begin{aligned}
\phi_{0}(t) \approx & 30+24.317 \cos (2 \pi \times 100 t)+2.702 \cos (6 \pi \times 100 t) \\
& +0.973 \cos (10 \pi \times 100 t)+0.496 \cos (14 \pi \times 100 t) \\
& +0.300 \times \cos (18 \pi \times 100 t)+0.201 \cos (22 \pi \times 100 t) \\
& +0.144 \cos (26 \pi \times 100 t)+0.108 \cos (30 \pi \times 100 t) \\
& +0.084 \cos (34 \pi \times 100 t)+0.067 \cos (38 \pi \times 100 t)
\end{aligned}
$$

With the above $\phi_{0}(t)$ in Eq. (20) and based on Equations (2)(5), two segments of SMI signals $g(t)$ are generated by incorporating two pairs of $C$ and $\alpha$ with $C / \alpha$ as $0.800 / 3.000$ 
and 5.000/5.000 respectively. Then the computer simulations are carried out according to the following:

Step 1: The fundamental angular frequency $\left(\omega_{0}\right)$ of the external vibration is estimated using the auto-correlation function of $g(t)$ [2];

Step 2: According to the selection rules presented in Section 2, $2 N+2$ data samples are selected respectively from a segment of an SMI signal $g(t)$ and its corresponding derivatives $g^{\prime}(t)$. Then all the coefficients in Eq. (18) are determined using Eq. (16);

Step 3: Eq (18) is solved to yield the parameters including $u_{1}$, $u_{2}$ and all Fourier coefficients $a_{1} \cdots a_{10}$ and $b_{1} \cdots b_{10}$;

Step 4: Eq. (15) is used to obtain $C$ and $\alpha$, and the vibration by Eq. (5) is reconstructed using the obtained coefficients.

The results of simulations are shown in Fig. 2, including $\phi_{0}(t)$ , $g(t)$, the reconstructed vibration trace (denoted by $\left.\tilde{\phi}_{0}(t)\right)$ and the error associated with the estimation. It is seen that, for both of the two cases, $\tilde{\phi}_{0}(t)$ is very close to $\phi_{0}(t)$, with the error less than $0.11 \%$ and $0.006 \%$ respectively, thus giving an accurate estimation of the vibration trace. In addition, the two parameters $C$ and $\alpha$ are also obtained with a reasonably high accuracy. For the case of $C / \alpha=0.800 / 3.000$, we obtained $C / \alpha$ with the accuracy of $0.5 \%$ and $1.9 \%$ and in the situation of strong feedback $C / \alpha=5.000 / 5.000$, we have obtained $C / \alpha$ with a accuracy of
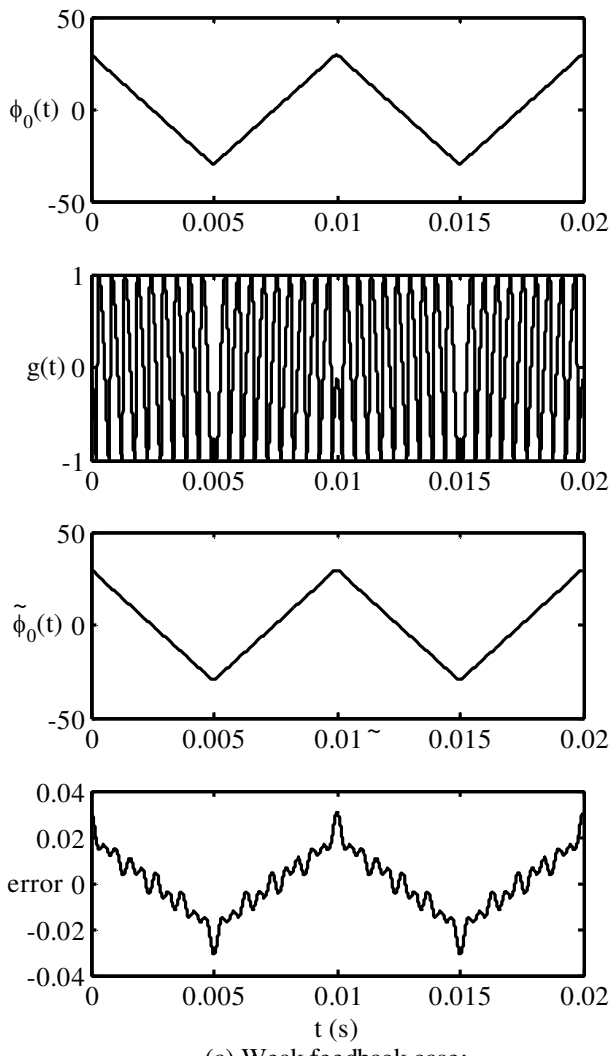

(a) Weak feedback case: $\mathrm{C} / \alpha=0.800 / 3.000 \quad \tilde{\mathrm{C}} / \tilde{\alpha}=0.796 / 3.057$
$0.06 \%$ and $0.12 \%$. Note that the value in accuracy for parameters estimation is the relative standard deviation of the estimated results with respect to true parameter values in the simulation.

The results show that, the proposed method is able to measure vibration and all the parameters simultaneously with reasonable estimation accuracy, under different feedback regime (week or strong), for a periodical vibration containing many harmonics.

\section{B. Experiment}

Experiments are also conducted to verify the effectiveness of the proposed method by means of the experimental SMI configuration illustrated in Fig. 3. A GaAlAs multi-quantum well (MQW) laser diode (LD) with module HL7851G from HITACHI is employed in our experiments. In the experiment, the $\mathrm{LD}$ is controlled by a laser controller (LDC2000) and a temperature controller (TEC200). We set the LD working at $140 \mathrm{~mA}$ (typical operating injection current) with an emitting wavelength of 785 $\mathrm{nm}$ and the operating temperature at $25^{\circ} \mathrm{C}$. A loudspeaker is used to form the external cavity with the initial cavity length of $310 \mathrm{~mm}\left(L_{0}\right)$. The loudspeaker is driven by a sinusoidal signal of $80 \mathrm{~Hz}$ and 6 Volt amplitude peak-to-peak. The vibrating target is kept unchanged through our experiments. Three experimental SMI signals with different optical feedback levels are obtained.
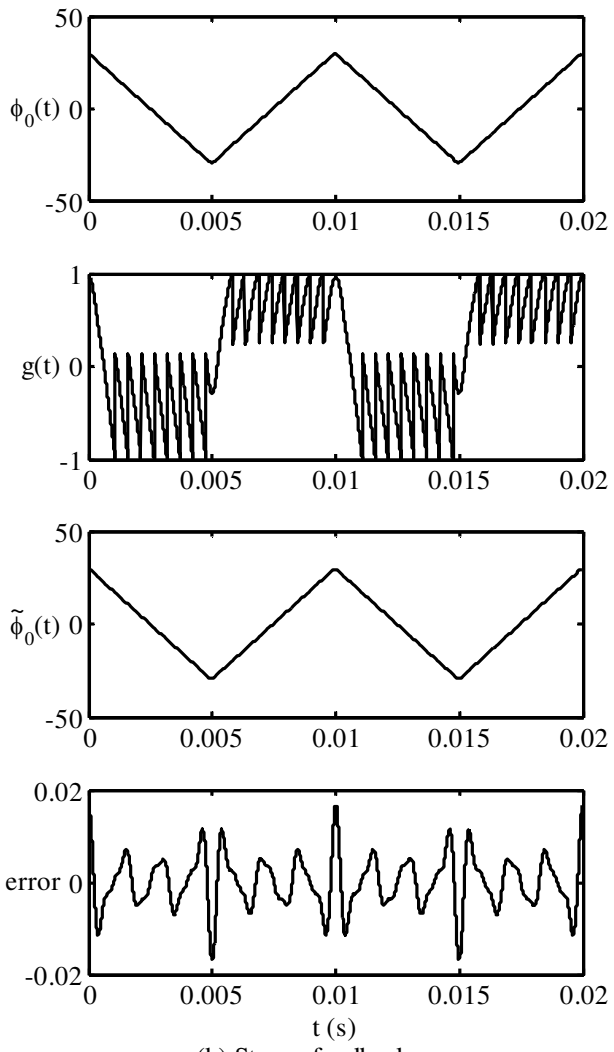

(b) Strong feedback case $\mathrm{C} / \alpha=5.000 / 5.000 \quad \tilde{\mathrm{C}} / \tilde{\alpha}=4.997 / 5.006$

Fig. 2. Reconstruction results for triangular vibration. (a) reconstruction results for weak feedback regime with true values: $C / \alpha=0.800 / 3.000 ;$ (b) reconstruction results for strong feedback regime with true values: $C / \alpha=5.000 / 5.000$. 


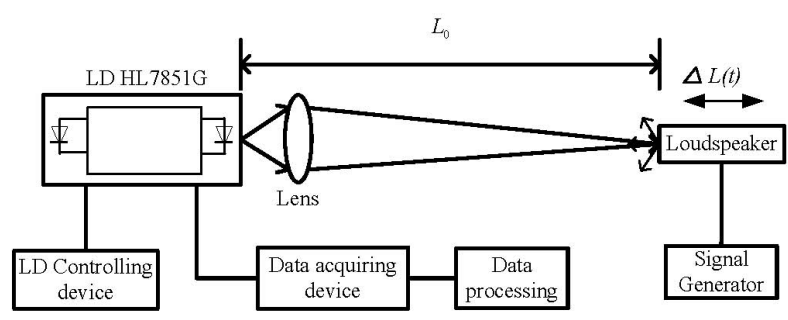

Fig. 3. SMI configuration

By considering 10-order Fourier extensions (it is enough to include the possible harmonics containing in the target vibration), we applied our algorithm on these further de-noised experimental signals using the method described in [16], then obtained parameters $C, \alpha$ and reconstructed the vibration of the loudspeaker. The results are shown in Fig. 4. The left column shows three SMI signals with different feedback levels. The right column shows the reconstructed vibration and the estimated values for $C$ and $\alpha$. It can be seen, firstly, the three reconstructed vibration are identical to each other as they correspond to same vibration. The magnitude of the vibration is with 1.83 um in average. This value has been confirmed by


(a) means of a commercial laser sensor LTC-025-04-SA from MTI instruments. The estimated values for both $C$ and $\alpha$ are also confirmed by our previous method presented in [7].

\section{Conclusion}

In this paper, we propose an algorithm to simultaneously retrieve the vibration and multiple parameters associated with SLs using an SMI sensing system. In contrast to the existing work on SMI based vibration measurement, the proposed method does not require the knowledge the $C$ and $\alpha$, and in comparison with existing approaches for $C$ and $\alpha$ measurement, the algorithm proposed does not need the detailed information on the movement trace of the object. Furthermore, the proposed method can work at any optical feedback regime. Therefore, the proposed algorithm lifts all the restrictions existed in SMI based sensing methods. Also, simple and low computation makes it easy to be implemented in practice. Both computer simulations and experiment have been used to verify the proposed algorithm, showing that the vibration, the optical feedback level and the alpha factor can be effectively estimated.
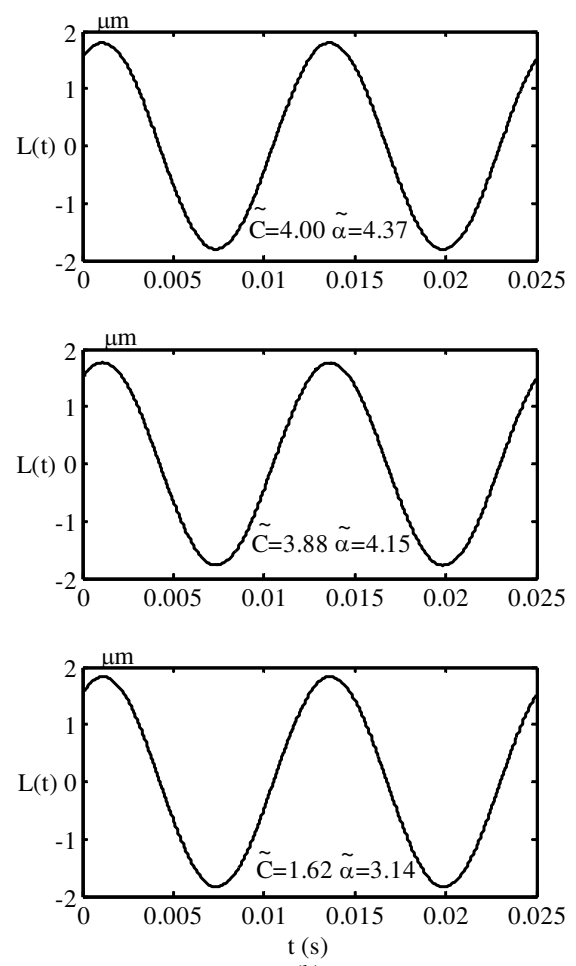

(b)

Fig. 4. Vibration reconstruction and parameters estimation results by using experimental data. (a) three SMI signals under different optical feedback regime; (b) vibration reconstruction and parameter estimation results

\section{Reference}

1. Y. Yu, G. Giuliani, and S. Donati, "Measurement of the linewidth enhancement factor of semiconductor lasers based on 
the optical feedback self-mixing effect," Photonics Technology Letters, IEEE 16, 990-992 (2004).

2. Y. Yu, J. Xi, J. Chicharo, and T. Bosch, "Toward Automatic Measurement of the Linewidth-Enhancement Factor Using Optical Feedback Self-Mixing Interferometry With Weak Optical Feedback," Quantum Electronics, IEEE Journal of 43, 527-534 (2007).

3. Y. Yu, J. Xi, J. Chicharo, and Y. Zhao, "A new approach for measuring the line-width enhancement factor," in International Conference on Intelligent Sensors, Sensor Networks and Information Processing, 2008. ISSNIP 2008. , 2008), 471-474.

4. W. Lu, J. Xi, Y. Yu, and J. Chicharo, "Linewidth enhancement factor measurement based on optical feedback selfmixing effect: a genetic algorithm approach," Journal of Optics A: Pure and Applied Optics 11, 045505 (2009).

5. Y. Fan, Y. Yu, J. Xi, and J. Chicharo, "Improving the measurement performance for a self-mixing interferometrybased displacement sensing system," Appl. Opt. 50, 5064-5072 (2011).

6. Y. Yu, J. Xi, and J. Chicharo, "Measuring the feedback parameter of a semiconductor laser with external optical feedback," Opt. Express 19, 9582-9593 (2011).

7. Y. Yu and J. Xi, "Influence of external optical feedback on the alpha factor of semiconductor lasers," Opt. Lett. 38, 17811783 (2013).

8. J. Xi, Y. Yu, J. Chicharo, and T. Bosch, "Estimating the parameters of semiconductor lasers based on weak optical feedback self-mixing interferometry," Quantum Electronics, IEEE Journal of 41, 1058-1064 (2005).

9. S. Donati, G. Giuliani, and S. Merlo, "Laser diode feedback interferometer for measurement of displacements without ambiguity," Quantum Electronics, IEEE Journal of 31, 113-119 (1995)

10. O. D. Bernal, U. Zabit, and T. Bosch, "Study of Laser Feedback Phase Under Self-Mixing Leading to Improved Phase Unwrapping for Vibration Sensing," Sensors Journal, IEEE 13, 4962-4971 (2013).

11. N. Servagent, F. Gouaux, and T. Bosch, "Measurements of displacement using the self-mixing interference in a laser diode," Journal of Optics 29, 168 (1998).

12. C. Bes, G. Plantier, and T. Bosch, "Displacement measurements using a self-mixing laser diode under moderate feedback," Instrumentation and Measurement, IEEE Transactions on 55, 1101-1105 (2006).

13. A. Doncescu, C. Bes, and T. Bosch, "Displacement Estimation with an Optical Feedback Interferometer using an Evolutionary Algorithm," in Sensors, 2007 IEEE, 2007), 382-386.

14. G. Giuliani, S. Bozzi-Pietra, and S. Donati, "Self-mixing laser diode vibrometer," Measurement Science and Technology 14, 24 (2003).

15. A. Magnani, A. Pesatori, and M. Norgia, "Self-mixing vibrometer with real-time digital signal elaboration," Appl. Optics 51, 5318-5325 (2012).

16. Y. Yu, J. Xi, and J. Chicharo, "Improving the Performance in an Optical feedback Self-mixing Interferometry System using Digital Signal Pre-processing," in IEEE
International Symposium on Intelligent Signal Processing, 2007. WISP 2007. , 2007), 1-6. 